\title{
A New Method to Determine the Electroweak Couplings of Individual Light Flavours at LEP
}

\author{
J. Letts \\ University of Bologna and INFN, Italy \\ P. Mättig \\ University of Dortmund, Germany
}

\begin{abstract}
A method is presented for determining the yields and properties of individual light quark flavours in $Z^{0}$ decays that is essentially free of detailed assumptions about hadronisation. The method uses an equation system with the number of events which are single and double tagged by high energy hadrons as inputs. In addition, $\mathrm{SU}(2)$ isospin symmetry and the flavour independence of QCD are used to derive general relations between hadron production from the various primary light quarks. Assuming the branching fractions $R_{q}$ of the $Z^{0}$ into down and strange quarks to be the same, five million hadronic $Z^{0}$ decays may allow a precisions of $\delta\left(R_{d}=R_{s}\right) /\left(R_{d}=R_{s}\right) \sim 0.05$ and $\delta A_{F B}(d=s) \sim$ $\delta A_{F B}(u) \sim 0.015$ for the corresponding asymmetries. The method can be extended to include somewhat more model dependent symmetries of hadron production, which then allows the electroweak observables for each of the individual light quarks to be determined.
\end{abstract}

(To be submitted to Z. Phys. C) 


\section{Introduction}

During the last two decades the Standard Model of electroweak interactions [1] has been confirmed experimentally to astounding precision. In particular the recent measurements at LEP and SLC of a large number of observables [2] have been a strong challenge to the Standard Model. In spite of the considerable effort and scrutiny there is no experimental data which disagrees significantly from theoretical expectations.

Still there are unattractive features of the Standard Model, such as its departure from the apparently most simple pattern. Among the more obvious ones is the proliferation of generations. Within the Standard Model, the elementary quarks and leptons are arranged in doublets. Their electroweak couplings to the $Z^{0}$ are determined by their weak isospins and electric charges. Neglecting colour degrees of freedom there are three generations which are made up of pairs of quarks and of leptons. The Standard Model has no explanation for their multiple existence and believes them to behave identically in strong and electroweak interactions. One of the experimental challenges of recent years has been to verify this conjecture.

Universal couplings of the various lepton species with respect to both charged [3] and neutral [2] weak currents have been confirmed to very high precision. Less stringent are the corresponding tests in the quark sector. Recent measurements of the flavour independence of the strong interactions support fermion universality [4] to within $\sim 10 \%$. The set of measurements of the $Z^{0}$ couplings to individual quark species is incomplete. Rather precise experimental results are available for the branching fractions and asymmetries of charm and especially beauty quarks [2]. The branching fractions in particular exhibit the largest deviations from theoretical expectations yet observed, there being too many beauty quarks produced and too few charm quarks. Although neither of these measurements is currently significant enough to claim a failure of the Standard Model, they have aroused a large interest and have been called the ' $R_{b} R_{c}$ ' crisis. Explanations that have been proposed include the existence of new quark species that mix with the standard ones [5] or separate $d$-type and $u$-type universal couplings [6]. Several of these attempts to explain the ' $R_{b} R_{c}$ ' crisis also imply non-Standard Model couplings in the light quark sector, where only few measurements exist. Some information on up and down quarks can be derived from neutrino scattering and atomic parity violation experiments which tend, interestingly enough, to deviate from the Standard Model expectations in a similar way as the beauty and charm measurements [7]. At LEP, the yield of photon radiation from quarks [8], which is dominated by quarks of electric charge $2 / 3$, also indicates a deficiency of uptype quarks. This underlines the need for complementary measurements of light quark electroweak observables.

In this paper we propose and evaluate a new method to determine the relative yields of light flavours. In determining the couplings of light quarks in $Z^{0}$ decays the main difficulty is their precise and unambiguous identification. Whereas both beauty and charm quarks have unique signatures and are essentially produced only as the primary quarks in $Z^{0}$ decays, light quarks frequently originate from the hadronisation process. This process is incalculable and therefore subject to considerable uncertainties. On the other hand its details may strongly affect the tagging efficiencies and purities of light quark samples and 
thus the extraction of their electroweak observables. It is the aim of this paper to suggest a way of minimising the reliance on models of the hadronisation mechanism. Our method makes use of the high statistics at LEP and tags the quarks by highly energetic particles which are assumed to have strong flavour and charge correlations to the primary quark.

One approach which can be used to estimate the flavour purity and tagging efficiency of such an event sample is to vary the parameters in a hadronisation model and estimate the impact on the required electroweak observables, as was chosen in reference [9]. Such an approach relies significantly on model ingredients that neither follow from basic principles of QCD nor are tightly constrained by data. Firstly, one has to assume a certain parametrisation of hadron production and secondly, one has to unfold the parameter values from the data. Finally, in doing so, assumptions have to be made about the relative yields of the various primary quark flavours. Even given the success of QCD shower models in describing many features of hadronic final states, the number of and correlations between assumptions is too large to test safely the fundamental properties of the electroweak model.

Our method almost completely avoids these uncertainties by using two ingredients. The first is the use of double tagged events with highly energetic particles in each of the event hemispheres to determine flavour tagging efficiencies and purities. The second is symmetries of the hadronisation mechanism which follow from $\mathrm{SU}(2)$ isospin symmetry and the flavour independence of QCD. These ingredients allow an equation system to be constructed which can be solved for the electroweak observables. Although we will show that the analysis can be based on data alone to a large extent, we will frequently make use of the JETSET model [10] to make some of our points more transparent.

In Section 2 we will outline the basic strategy of the measurements of the light flavour widths and asymmetries. Before developing these in more detail in Sections 4 and 5, we will show first in Section 3 that heavy flavour backgrounds can be easily determined. Potential sources of error of the method are discussed in section 6 and finally the method is applied to a sample of Monte Carlo events in Section 7.

\section{The General Strategy}

The basic electroweak observables for quarks at LEP are the partial widths and the forward-backward asymmetries. The partial widths $\Gamma_{q \bar{q}}$ of the $Z^{0}$ into a quark species $q$, are given by

$$
\Gamma_{q \bar{q}} \propto v_{q}^{2}+a_{q}^{2} \propto \frac{N_{q}}{\mathcal{L}},
$$

where $v_{q}$ and $a_{q}$ are the vector and axial couplings of the quark type $q, N_{q}$ is the number of $Z^{0}$ decays into quark type $q$ and $\mathcal{L}$ is the collected luminosity. Instead of the partial width we will use the decay branching fraction

$$
R_{q}=\frac{\Gamma_{q \bar{q}}}{\Gamma_{h a d}}
$$


where $\Gamma_{\text {had }}$ is the total hadronic width of the $Z^{0}$. The forward-backward asymmetry is given by

$$
A_{F B}(q)=\frac{N_{q}(\cos \theta>0)-N_{q}(\cos \theta<0)}{N_{q}} \propto\left(v_{q} / a_{q}\right) /\left(1+\left(v_{q} / a_{q}\right)^{2}\right),
$$

where $\theta$ is the scattering angle of the quark with respect to the incoming electron.

The main issue in measuring these observables for quarks is to find an efficient and well understood method of identifying the various species. This is rather easy for beauty quarks, which cannot only be tagged with purities of more than $90 \%$, but whose efficiency can be obtained from data with high precision using double tagged events in which a beauty particle is identified in each of the two event hemispheres [11]. To recall the important points, single tags suffer from systematic uncertainties in determining the tagging efficiency, $\epsilon_{b}$. Neglecting background,

$$
R_{b}=\frac{\Gamma_{b \bar{b}}}{\Gamma_{h a d}}=\frac{1}{\epsilon_{b}} \frac{N_{b}^{\text {tag }}}{N_{h a d}},
$$

which limits the uncertainty in $R_{b}$ to the uncertainty in the tagging efficiency $\mathcal{O}\left(\delta \epsilon_{b} / \epsilon_{b}\right) \sim$ $5-10 \%$. The uncertainties can be reduced by determining the tagging efficiency directly from the data by measuring the ratio of double to single tagged events,

$$
N_{b}^{t a g}=\epsilon_{b} \cdot 2 N_{Z^{0} \rightarrow b \bar{b}} ; \quad N_{b b}^{t a g}=\left(\epsilon_{b}\right)^{2} N_{Z^{0} \rightarrow b \bar{b}} \Rightarrow \epsilon_{b}=2 \frac{N_{b b}^{t a g}}{N_{b}^{t a g}} .
$$

This allows a measurement of $R_{b}$ which is dominated by the statistical uncertainty $\delta \epsilon_{b} / \epsilon_{b} \propto$ $1 / \sqrt{N_{b b}^{t a g}}$, at least for precisions down to $\sim 1 \%$. This method works so well because beauty events can be isolated by lifetime or lepton tagging with small, well understood backgrounds from other flavours and with a reasonably high efficiency.

Similarly one can obtain a sample of light flavours, up, down, and strange with little background from charm and beauty quarks by using stable or long-lived particles with high scaled momentum $x_{p}=2 p_{h} / E_{c m}>x_{c u t}$ as a tag [12]. Here $p_{h}$ is the momentum of hadron, $h$, and $E_{c m}$ the centre-of-mass energy. Particle species which are easy to measure are, for example, charged pions, charged kaons, and $K_{S}^{0}$. In Fig. 1 the tagging efficiencies for various flavours using different tagging particles are shown as a function of $x_{c u t}$, as predicted by the JETSET model. For high $x_{c u t}>0.4$ the efficiencies are a few percent and decrease by some $20-50 \%$ if $x_{c u t}$ is increased by 0.1 . Given the $4-5$ million multihadronic $Z^{0}$ decays available to each LEP experiment, the efficiencies of $\epsilon_{\text {tag }} \sim 10(2) \%$ for $x_{c u t}=0.4(0.6)$ imply some $400,000(80,000)$ tagged events, sufficient for our method.

The essential difference from the measurement involving beauty quarks is that for light quarks one does not deal with just one flavour but with an unknown mixture of at least three flavours. Whereas it is straightforward to determine electroweak parameters for such an inclusive sample, it is much more involved to disentangle the contributions of the individual light flavours. Firstly, it requires one to identify the different particle types. This has been shown to be possible at LEP through the use of $\mathrm{dE} / \mathrm{dx}$ measurements 
in tracking devices or Ring-Imaging Cerenkov counters [13], even for large values of $x_{p}$. Secondly, one has to find a way of estimating the relative flavour contributions in these samples. This is the main point of this article.

The general program to achieve this goal can be summarised as follows. As for beauty quarks, double tagging is the key to measure the electroweak properties of the individual light flavours. To determine the contributions of the different flavours, several tags with different sensitivity to the various flavours have to be used. The tagging efficiencies and relations between the various tags can be obtained from double tagged events. In addition, to disentangle these contributions, some basic symmetries of the hadronisation process have to be used.

Directly observable are the number of tags $N_{h}$ of a particle type $h$ and their forwardbackward charge asymmetries, $A_{F B}^{h}$. These measurable quantities are related to the $R_{q}$ by the fractions of event hemispheres with a primary quark $q$ which are tagged by a hadron $h$, denoted as $\eta_{q}^{h}$. Assume for definiteness a tagging particle of charged kaons. One then obtains a relation between the measured particle yields and the branching fractions:

$$
\frac{N_{K^{ \pm}}}{N_{h a d}}=2 \cdot\left(R_{d} \eta_{d}^{K^{ \pm}}+R_{u} \eta_{u}^{K^{ \pm}}+R_{s} \eta_{s}^{K^{ \pm}}+R_{c} \eta_{c}^{K^{ \pm}}+R_{b} \eta_{b}^{K^{ \pm}}\right)=2 \sum_{q} R_{q} \eta_{q}^{K^{ \pm}} .
$$

Therefore, to determine the $R_{q}$, the $\eta_{q}^{h}$ have to be known. Similarly, the forward-backward asymmetry is given by

$$
A_{F B}^{K^{ \pm}}=\sum_{q}\left\{s_{q} \cdot f_{q}^{K^{ \pm}} \cdot\left(2 r_{q}^{K^{ \pm}}-1\right)\right\} \cdot A_{F B}(q)=\sum_{q} F_{q}^{h} \cdot A_{F B}(q)
$$

where the $F_{q}^{h}$ are the coefficients needed to transform the measured hadron forwardbackward asymmetries into the quark asymmetries. The reliabilities, $r_{q}^{h}$, take into account dilutions due to the misidentification of the sign of the charges of the quarks, $s_{q}$, and are given by

$$
r_{q}^{h}=\frac{\left(N_{q}^{h}\right)^{c}}{\left(N_{q}^{h}\right)^{c}+\left(N_{q}^{h}\right)^{w}}=\frac{\left(N_{q}^{h}\right)^{c}}{N_{q}^{h}},
$$

where " $c$ " denotes the correct charge assignment and " $w$ " the wrong one. The fractions of flavour $q$ in the tagged kaon sample are given by

$$
f_{q}^{K^{ \pm}}=\frac{2 R_{q} \eta_{q}^{K^{ \pm}}}{N_{K^{ \pm}} / N_{h a d}}=\frac{R_{q} \eta_{q}^{K^{ \pm}}}{\left(\sum_{q^{\prime}} R_{q^{\prime}} \eta_{q^{\prime}}^{K^{ \pm}}\right)} .
$$

Thus, for the asymmetries, the reliabilities $r_{q}^{h}$ must be determined in addition to the $\eta_{q}^{h}$. We will start by discussing ways of determining the $\eta_{q}^{h}$, therefore allowing the partial widths to be measured. The method will then be extended to the asymmetries.

\section{Determining the Fraction of Heavy Flavours}

As a first step, we discuss how the contributions from charm and beauty quarks can be estimated such that the discussion can be restricted to the light flavours alone. In 
general, due to their high decay multiplicities, charm and beauty events are not expected to contribute much to a sample of events with high $x_{p}$ tags. If required, their fractions may be reduced event further by rejecting all events with a secondary vertex displaced from the interaction point, for example ${ }^{1}$. Efficient and well established procedures exist to tag charm and beauty events. These can be used to determine the remaining $f_{c}^{h}$ and $f_{b}^{h}$ by employing double tagged events with a high $x_{p}$ tag in one hemisphere and a heavy flavour tag in the opposite hemisphere. Since the heavy flavour tagging efficiencies are well known, the observed numbers of events can immediately be interpreted in terms of $f_{c}^{h}$ and $f_{b}^{h}$. If the charge sign of the heavy flavour tag is known, the reliabilities $r_{c}^{h}$ and $r_{b}^{h}$ can also be determined ${ }^{2}$.

In the case of beauty quarks, either lifetime tags or charged lepton tags can be used. Lifetime tags yield directly the $f_{b}^{h}$, whereas with lepton tags one can also obtain the reliabilities $r_{b}^{h}$. The JETSET model predicts that $5.6 \%$ of all hadrons with an $x_{\text {cut }}=0.4$ originate from beauty events. Given the high efficiency of the $b$ flavour tags, the $f_{b}^{h}$ and $r_{b}^{h}$ should be measurable to precisions of $\delta f_{b}^{h} \sim 0.5 \%$ and $\delta r_{b}^{h} \sim 10 \%$. Taking into account the mixing in the $B^{0}$ system, parametrised by the inclusive mixing parameter $\chi_{l}$, the reliability is given by

$$
r_{b}^{h}=\frac{V_{b}^{\ell, h}-\chi_{\ell}}{1-2 \chi_{\ell}},
$$

where $V_{b}^{\ell, h}$ is the fraction of all lepton - $h$ double tagged events with different charge signs. The parameter $\chi_{l}$ has been precisely measured at LEP to be $0.1147 \pm 0.0062[2]$.

A somewhat larger background in the light flavour sample is due to charm. Since charm hadrons have a lower decay product multiplicity, more stable high $x_{p}$ hadrons can be found. For example, the JETSET model predicts that $f_{c}^{h} \sim 10.9 \%$ for an $x_{\text {cut }}=0.4$. The cleanest way to tag charm is by using high momentum $D^{* \pm}$ mesons as a tag, which allows a charm purity of $\sim 80 \%$ for $x_{p}^{D^{* \pm}}>0.5$ to be obtained [15]. Alternatively, one may base the estimate on the measured production and decay properties of charmed hadrons. In this case the limiting factor may be the knowledge of the decay branching fraction of $D$ mesons into $K_{S}^{0}$ vs. $K^{ \pm}$, which has been measured to an accuracy of $\sim 10 \%$ [16].

In what follows we will assume that the $c$ and $b$ contributions are known and only the light quark partial widths, flavour fractions, and reliabilities need to be determined. Therefore, in the following sections the heavy flavour component is neglected.

\section{Determining the Partial Widths of Light Flavours}

\subsection{General Constraints}

In analogy to the determination of the partial width of beauty quarks, double tagged events can be used to determine the tagging efficiency for light quark events. Assuming

\footnotetext{
${ }^{1}$ We are grateful to M. Bobinski for suggesting this possibility.

${ }^{2}$ For this section on heavy flavour backgrounds, we profited largely from work done by C. Grandi [14].
} 
again for definiteness a tag of charged kaons, double tagged events occur with a frequency

$$
\frac{N_{K K}}{N_{h a d}}=\sum_{q} R_{q}\left(\eta_{q}^{K^{ \pm}}\right)^{2},
$$

where $N_{K K}$ stands for the number of double tagged charged kaon events in which the kaons can have any relative charges. Here we have assumed that there are no correlations between the tagging efficiencies of opposite event hemispheres. Although most kaons are thought to originate from strange quark events, the two measurements of single and double tagged kaons are not sufficient to determine $R_{s}$ because of the unknown and sizable contributions from up and down quarks. On the other hand, primary strange quarks are not supposed to hadronise frequently into pions, which are thought to be the dominant hadrons from up and down quark events. Thus, one can also use the information from pion tags:

$$
\frac{N_{\pi^{ \pm}}}{N_{h a d}}=2 \sum_{q} R_{q} \eta_{q}^{\pi^{ \pm}}, \frac{N_{\pi \pi}}{N_{h a d}}=\sum_{q} R_{q}\left(\eta_{q}^{\pi^{ \pm}}\right)^{2},
$$

and also the mixed term

$$
\frac{N_{\pi K}}{N_{h a d}}=2 \sum_{q} R_{q} \eta_{q}^{\pi^{ \pm}} \eta_{q}^{K^{ \pm}}
$$

Using various tags with different flavour tagging efficiencies leads to equations of the general type:

$$
\frac{N_{h}}{N_{h a d}}=2 \sum_{q} R_{q} \eta_{q}^{h}, \frac{N_{k l}}{N_{h a d}}=\left(2-\delta_{k l}\right) \sum_{q} R_{q} \eta_{q}^{k} \eta_{q}^{l}
$$

where $\delta_{k l}=0$ for $k \neq l$ and $\delta_{k l}=1$ for $k=l$. This leads to a system of coupled non-linear equations:

$$
\frac{1}{N_{h a d}}\left(\begin{array}{c}
\frac{1}{2} N_{h} \\
\vdots \\
\frac{1}{2-\delta_{k l}} N_{k l} \\
\vdots
\end{array}\right)=\left(\begin{array}{ccc}
\eta_{u}^{h} & \eta_{d}^{h} & \eta_{s}^{h} \\
\vdots & \vdots & \vdots \\
\eta_{u}^{k} \eta_{u}^{l} & \eta_{d}^{k} \eta_{d}^{l} & \eta_{s}^{k} \eta_{s}^{l} \\
\vdots & \vdots & \vdots
\end{array}\right)\left(\begin{array}{c}
R_{u} \\
R_{d} \\
R_{s}
\end{array}\right) .
$$

Assuming that there are $n$ hadron types available as tagging particles, one obtains $\left(n^{2}+\right.$ $n) / 2$ double tagged combinations and $n$ single tags, giving a total of $\left(n^{2}+3 n\right) / 2$ measurements with which to determine the $3 n$ unknown $\eta_{q}^{h}$ and the 3 unknown light flavour $R_{q}$.

In principle all hadrons consisting of light quarks can be used as tagging particles. For example, $\Delta^{-}$and $\Delta^{++}$would be excellent to identify down and up quarks, respectively. Similarly the $\phi(1020)$ meson would be ideal to tag strange quark events. However, it is experimentally preferable to use those hadrons which can be identified in great numbers with high purity at large $x_{p}$. The most promising candidates fulfilling these requirements are $\pi^{ \pm}, K^{ \pm}, K_{S}^{0}$, and protons. We will evaluate the model based on these particles. However, in an experimental analysis additional tagging particle types such as $\Lambda$ or $\pi^{0}$ may be useful as light flavour tags, depending on the efficiency and purity with which they can be identified. It should be noted that the meson triplet we do use is well suited for our purposes, since their quark contents combine the light quark species in all three possible combinations $(u, d),(u, s)$, and $(d, s)$. The protons should predominantly originate from up quarks. However, since the production mechanism for leading protons 
is not well understood, it is not clear to what extent they reflect the quantum numbers of the primary quark. Using the four tagging particle types we have chosen for our example, 14 measurements can be made in order to determine 12 unknown $\eta_{q}^{h}$ and the three unknown light flavours $R_{q}$. Therefore, the equation system is under-constrained. Since the unknowns of the system appear as products $R_{q} \eta_{q}^{h}$, the number of required additional constraints is not defined in an unambiguous way, but depends on the kind of constraints. Assuming, for example, that all $R_{q}$ are unknown, we find that the system can be solved for $n$ additional unknowns, $n$ being the number of hadron species considered. Thus, additional constraints on the $\eta_{q}^{h}$ have to be found. Alternatively, one can assume relations among the $R_{q}$, which simplifies the equation system and reduced the number of additional constraints on the $\eta_{q}^{h}$ which are needed. In the following section we will discuss possible relations.

Some constraints follow directly from normalisation properties. The total hadronic width of the $Z^{0}$ implies that:

$$
R_{\text {light }}=R_{d}+R_{u}+R_{s}=1-R_{b}-R_{c}
$$

such that $R_{\text {light }}$ can be based on the well measured fractions $R_{b}$ and $R_{c}$.

To obtain a stable, physical solution of the non-linear equation system, one must invoke at least one general additional assumption about hadronisation since the solution of the equation system is ambiguous. This ambiguity arises since none of these equations implies a preference of a quark type $q$ to yield a tagging hadron of type $h$. For example, the equations are symmetric for a preference of a strange quark to fragment into either a pion or a kaon. This ambiguity can be resolved by the relations

$$
\eta_{s}^{K^{ \pm}}>\eta_{u}^{K^{ \pm}}>\eta_{d}^{K^{ \pm}} ; \eta_{d}^{\pi^{ \pm}}, \eta_{u}^{\pi^{ \pm}}>\eta_{s}^{\pi^{ \pm}}, \text {etc. }
$$

The underlying physics of these inequalities is the bremsstrahlung character of gluon radiation which leaves most of the energy with the radiator. They are experimentally supported by the observations of long range charge correlations in jets [17], which implies that at least the electric charge of the quark manifests itself in the charged particles of highest energy. The above inequalities may be directly tested in the future using $W^{ \pm}$decays by studying decays into charm and strange quarks, for example.

\subsection{Symmetries in Hadronisation}

Assuming that measurements can be performed only with the statistically most interesting particle species, charged pions, charged kaons, neutral kaons, and protons, additional constraints must be found in order to determine the light flavour partial widths. The missing constraints can be based on fundamental symmetries of the hadronisation process. It is a general assumption of hadronisation models that mesons are formed from a (primary or sea) quark $q$ which combines with an antiquark $q^{\prime}$ from the sea (or the charge conjugate process). Models such as cluster fragmentation [18], as implemented in the HERWIG [19] Monte Carlo, and string fragmentation [20], as in JETSET, have been developed to describe this process. Our basic argument applies to both, but for definiteness we will argue along lines of the string model. 
Consider for a moment only stable hadrons $h$ which have a quark content $\left(q q^{\prime}\right)$ and are directly produced from primary quarks of flavour $q$. Then one can schematically relate the $\eta_{q}^{h}$ to the hadronisation properties by

$$
\eta_{q}^{h=\left(q q^{\prime}\right)}\left(x_{c u t}\right)=\gamma_{q^{\prime}} \int_{x_{c u t}}^{1} \mathrm{D}_{q}^{h}\left(x_{p}\right) d x=\gamma_{q^{\prime}} \mathcal{D}_{q}^{h}\left(x_{c u t}\right)
$$

where $\mathrm{D}_{q}^{h}\left(x_{p}\right)$ describes the shape of the fragmentation function and $\gamma_{q^{\prime}}$ is the probability for a quark of flavour $q^{\prime}$ to pop out of the hadronisation sea.

Motivated by the flavour independence of strong interactions [4], the $\gamma_{q}$ should be independent of the flavour of the primary quark. Thus, for example, a strange quark should be picked up as frequently from a primary up quark as from a down quark. Less certain are the actual values of the $\gamma_{q}$, which are assumed to be strongly dependent on the quark mass. Because of the high charm and beauty masses, $\gamma_{b}$ and $\gamma_{c}$ are negligible and only light quarks contribute to the sea, a supposition which is supported by recent measurements [21]. Another rather safe assumption is that SU(2) isospin symmetry implies that $\gamma_{d}=\gamma_{u}$, a conjecture supported by LEP measurements of hadron production rates [22]. The value of $\gamma_{s}$ cannot be obtained from such a symmetry. Several measurements indicate that $2 \gamma_{s} /\left(\gamma_{u}+\gamma_{d}\right) \sim 0.29 \pm \mathbf{0 . 0 1 5}[23]$. Such simple suppression factors do not seem to describe the production of baryons, which to date has posed a particular problem for hadronisation models.

Apart from the relative yields of hadron species, the shapes of the fragmentation functions $\mathrm{D}_{q}^{h}\left(x_{p}\right)$ are uncertain and may depend on the hadron mass. Assuming $\mathrm{SU}(2)$ isospin symmetry, $\mathcal{D}_{q}^{h}\left(x_{c u t}\right)$ should be identical for $K^{ \pm}$and $K^{0}$, whereas a firm relation between $\mathcal{D}_{q}^{K^{ \pm}}\left(x_{\text {cut }}\right)$ and $\mathcal{D}_{q}^{\pi^{ \pm}}\left(x_{\text {cut }}\right)$ cannot be derived.

In reality this pattern is more complicated since direct production of hadrons from primary quarks, to be denoted by $P_{q}^{h}$, is not the only source of tagging particles. High momentum hadrons are also produced from the decays of resonances which contain the primary quarks (denoted by $Z_{q}^{h}$ ) and from the non-primary hadronisation (denoted by $\left.H_{q}^{h}\right)$. Therefore,

$$
\eta_{q}^{h}\left(x_{\text {cut }}\right) \propto P_{q}^{h}\left(x_{\text {cut }}\right)+Z_{q}^{h}\left(x_{\text {cut }}\right)+H_{q}^{h}\left(x_{\text {cut }}\right) .
$$

These contributions have two important effects in that they tend to mix the $\eta_{q}^{h}$ from the various quark species and that they can potentially break some of the hadronisation symmetry relations. One should note that, whereas the $Z_{q}^{h}$ contribution still has some relation to the primary quark, e.g. a $K^{*}(892)$ which contains a primary strange quark still decays into a strange particle, the $H_{q}^{h}$ contribution is identical for all primary flavours and is charge symmetric, apart from small effects due to masses and perhaps baryon production. The hadronisation component therefore reduces the discrimination power among the various flavours. In Fig. 2 we show the contributions of the various sources for the production of charged kaons in strange quark events as expected by the JETSET model. As is clearly seen, decay products are predominant at low momenta and make an important contribution over a large range of $x_{c u t}$. The hadronisation contribution, $H_{q}^{h}$, is particularly important at very low $x_{c u t}$ and rapidly looses importance for high $x_{c u t}$. The detailed fractions depend on the particle type and the quark flavour; however, the general 
trend remains that the higher $x_{c u t}$ one chooses, the less the decay and hadronisation particles contribute to the tagged sample.

To understand the mixing between the flavours due to decays $Z_{q}^{h}$ one can consider again the case of $K^{*}(892)$ production. According to the above arguments, $P_{d}^{K^{ \pm}}=0$ since charged kaons contain no $d$ valence quarks, implying that direct primary kaons are only due to up and strange quark events. However, $K^{*}(892)^{0}$ production from a primary down quark and the subsequent decay $K^{*}(892)^{0} \rightarrow K^{+} \pi^{-}$makes it possible that $\eta_{d}^{K^{ \pm}} \neq 0$. This effect reduces the discrimination power of the different particle species by introducing correlations between the derived flavour contributions, thus diluting the determination of the partial widths. Correlations between the various $R_{q}$ can be minimised by optimising the selection criteria such that one hadron type $h$ originates mainly from one flavour, i.e. $\eta_{q}^{h} /\left(\sum_{q^{\prime}} \eta_{q^{\prime}}^{h}\right)$ is large. Thus, preferentially only "first-rank" and directly produced particles should be tagged, i.e. $Z_{q}^{h}, H_{q}^{h} \rightarrow 0$. This is the basic argument to select only particles with high $x_{p}$.

The above considerations lead to the following hierarchy for choosing additional constraints on the particle contributions:

- The safest approach is to avoid any reliance on the absolute value of any of the $\gamma_{q}$.

- Relations based on $\mathrm{SU}(2)$ isospin symmetry, i.e. $\gamma_{u} \sim \gamma_{d}$, are rather well motivated.

- If required, a value of $2 \gamma_{s} /\left(\gamma_{d}+\gamma_{u}\right)$ can be used. However, one should allow for a reasonably large variation of this parameter to account for potential systematic uncertainties.

- Relations involving baryons seem rather uncertain and should be avoided.

\subsection{Examples of Hadronisation Symmetry Relations}

Rather precise relations which survive the most important decays and hadronisation effects, modulo tiny mass effects, are those which are totally free of any dependence on the value of any of the $\gamma_{q}$ :

$$
\begin{aligned}
& \eta_{d}^{K^{0}}=\eta_{u}^{K^{ \pm}}, \\
& \eta_{u}^{K^{0}}=\eta_{d}^{K^{ \pm}} .
\end{aligned}
$$

It should be noted that here $K^{0}$ implies $K_{S}^{0}$ plus $K_{L}^{0}$. A relation which is exactly true only if $\gamma_{u}=\gamma_{d}$ is

$$
\eta_{d}^{\pi^{ \pm}}=\eta_{u}^{\pi^{ \pm}}
$$

Similarly one can form a relation for kaon production from strange quark events:

$$
\eta_{s}^{K^{ \pm}}=\eta_{s}^{K^{0}}
$$

If $\gamma_{u} \neq \gamma_{d}$ the appropriate ratio has to be included, e.g. to first order $\eta_{d}^{\pi^{ \pm}}=\frac{\gamma_{u}}{\gamma_{d}} \eta_{u}^{\pi^{ \pm}}$. Decays may in principle complicate these relations further. For example, since pions can 
also be produced in $K^{*}(892)$ decays, some additional contribution proportional to $\gamma_{s}$ has to be taken into account in Eq. 22. Equation 23 can be broken by $\phi(1020)$ decays since these are not symmetric with respect to $K^{0} \leftrightarrow K^{ \pm}$. The two latter relations are therefore exact only in the limit when only the $P_{a}^{h}$ component contributes to hadron production. As discussed before, this can be fairly well approximated by choosing a high $x_{c u t}$ and allowing for a reasonable breaking of the symmetries when calculating the systematic errors. As an example we show the validity of the hadronisation symmetries given above as expected from the JETSET model in Fig. 3. Whereas some deviations exist for lower scaled momenta $x_{p}$, no statistically significant deviation of more than $2 \%$ is observed for $x_{p}>0.3$. In the subsequent discussion, we will assume that $\mathrm{SU}(2)$ isospin symmetry holds.

For four kinds of particle tags, these hadronisation symmetries leave one with eight unknown efficiencies $\eta_{q}^{h}$ and two unknown $R_{q}$ 's, taking into account the constraint from $R_{b}$ and $R_{c}$. According to the preceding discussion, this is not sufficient to solve the equation system. There are two possible solutions to this problem. The first is to introduce additional hadronisation relations, perhaps at the cost of increased model dependence, while the second is to have a less ambitious physics goal.

A close inspection of the equation system reveals that one major challenge is the separation of up and down quarks. Whereas the strange quarks are tagged predominantly by charged and neutral kaons, the separation of up and down production is essentially given by the difference of the charged and neutral kaon yields, which is rather small. Therefore, a hadronisation relation between kaons and pions, or between strange and either up or down quarks would be of particular help. In the following we describe some possible additional hadronisation relations.

It is suggestive to assume that $\eta_{s}^{K^{ \pm}}=\eta_{d}^{\pi^{ \pm}}$, which would be true if all pion and kaon production were primary and also if the shape of the pion and kaon fragmentation functions were identical. However, there is no theoretical justification for such assumptions. Instead, for example, the LUND symmetric fragmentation function [24] predicts that the kaon fragmentation function is harder than the one for pions, implying some $x_{p}$ dependent proportionality $\eta_{s}^{K^{ \pm}}\left(x_{p}\right)=\gamma_{\pi}\left(x_{p}\right) \cdot \eta_{d}^{\pi}\left(x_{p}\right)$. In addition, the relation would also be broken by the contributions from decays and hadronisation, $Z_{q}^{h}$ and $H_{q}^{h}$. Similar arguments apply to relations such as $\eta_{u}^{K^{ \pm}}=\gamma_{s} \cdot \eta_{u}^{\pi^{ \pm}}$. Invoking these relations therefore implies some model dependence. Without going into too much detail, let us point out that the model dependence may be reduced by tests with the data. For example, the primary component $P_{q}^{h}$ may be disentangled from the hadronisation component $H_{q}^{h}$ by exploiting the property that primary hadrons have opposite electric charges in opposite hemispheres. A study of double tagged events may therefore lead to important constraints on the $x_{p}$ dependence of the $P_{q}^{\pi}$ and $P_{q}^{K}$ components. Otherwise, contributions to $Z_{q}^{h}$ from $\rho(770)$ or $K^{*}(892)$ decays can be estimated from their measured production rates. Therefore, it may be possible to constrain $\gamma_{\pi}\left(x_{p}\right)$ from the data.

Given the general agreement of many features of the JETSET model with data, one can go a step further and use the model to find additional relations with rather good 
stability with respect to $x_{c u t}$. Two examples are shown in Fig. 4. The ratio

$$
\left(\eta_{s}^{\pi^{ \pm}}+\eta_{s}^{K^{ \pm}}\right) /\left(\eta_{d}^{\pi^{ \pm}}+\eta_{d}^{K^{ \pm}}\right)
$$

is close to one for the interesting range $0.3<x_{\text {cut }}<0.6$. Outside this range the relation is broken. For $x_{c u t} \rightarrow 1$ the ratio should approach $\eta_{s}^{K^{ \pm}} / \eta_{d}^{\pi^{ \pm}}$, which may be $>1$ due to the assumed harder kaon fragmentation function, while for low values of $x_{c u t}$ the pion yield from the $Z_{q}^{h}$ component leads to a decrease. At intermediate values of $x_{c u t}$ this relation is an expression of the equal probability for $d$ and $s$ quarks to be tagged by a stable charged meson.

Another quite stable relation is

$$
\left(\eta_{d}^{K^{ \pm}}+\eta_{u}^{K^{ \pm}}\right) / \eta_{u}^{\pi^{ \pm}}
$$

with a value of $\approx 0.38$. At high values of $x_{c u t}$ the ratio is essentially given by $\eta_{u}^{K^{ \pm}} / \eta_{u}^{\pi^{ \pm}}$. At lower values of $x_{c u t}$ the $H_{q}^{h}$ and $Z_{q}^{h}$ components become more important. As can be seen in Fig. 5, the relation $\eta_{u}^{K^{ \pm}} / \eta_{u}^{\pi^{ \pm}}$is not constant due to a sizable contribution from $u \rightarrow K^{*}(892) \rightarrow K \pi$ in which the kaon is not the higher momentum hadron. This effect is mostly compensated by the inclusion of $\eta_{d}^{K^{ \pm}}=\eta_{u}^{K^{0}}$ in the ratio. We conclude that both of the relations in Eqs. 24 and 25 exhibit nice features for our method. However, these properties are due to compensations of various effects, which makes them model dependent, although this dependence may be reduced by further studies. The model dependence of these relations will be discussed in Section 6 .

In the following we choose the alternative approach of having a less ambitious physics goal by assuming a relation between the branching fractions $R_{q}$. Motivated by the assumed equality of up-type and down-type quark couplings in the Standard Model, we impose the constraint $R_{d}=R_{s}$. As will be shown in Section 7, this constraint yields the desired stability even with the statistics available.

\section{$5 \quad$ Light Flavour Asymmetries}

The knowledge of the flavour fractions $f_{q}^{h}=2 R_{q} \eta_{q}^{h} /\left(N_{h} / N_{h a d}\right)$ can next be used to disentangle the hadron asymmetries to find the flavour asymmetries. One new aspect, however, is that only baryons or charged mesons can be used. The $K_{S}^{0}$ does not carry any direct information about the asymmetries of light flavours, since it cannot tag the charge of the primary quark. Another new requirement for the asymmetry measurement is that the sign of the charge of the tagging hadron ${ }^{3}$ should coincide with the sign of the charge of the primary quark. Non-primary sources of hadron production lead to dilutions of the measured asymmetries. For example, a primary $d$ quark can form a first-rank $\rho(770)^{0}$, which then decays to $\pi^{+} \pi^{-}$. In half of the decays the $\pi^{+}$has a larger momentum and may be tagged, although the charge of the $d$ quark is carried by the $\pi^{-}$.

The dilution due to these effects may be parametrised by a reliability, $r_{q}^{h}$, which was defined in Section 2. Therefore, as given in Section 2, the coefficients $F_{q}^{h}$ needed to

\footnotetext{
${ }^{3}$ For baryons it is the baryon number.
} 
transform the observed hadron asymmetries into the flavour asymmetries via

$$
A_{F B}^{h}=\sum_{q} F_{q}^{h} \cdot A_{F B}(q)
$$

are given by

$$
F_{q}^{h}=s_{q} \cdot f_{q}^{h} \cdot\left(2 r_{q}^{h}-1\right)=s_{q} \cdot\left\{\frac{2 R_{q} \eta_{q}^{h}}{N_{h} / N_{h a d}}\right\} \cdot\left(2 r_{q}^{h}-1\right) .
$$

In addition to the $R_{q}$ and $\eta_{q}^{h}$, whose determination has been discussed in the preceding section, the $r_{q}^{h}$ must also be determined.

Directly observable experimentally are the flavour integrated reliabilities for the various hadron species as given by the charge correlations of double tagged events with particle types $k$ and $l$ :

$$
\begin{aligned}
V^{k, l} & =\frac{N^{k^{ \pm} l^{\mp}}}{N^{k^{ \pm} l^{\mp}}+N^{k^{ \pm} l^{ \pm}}} \\
& =\sum_{q} \frac{\eta_{q}^{k} \cdot \eta_{q}^{l} \cdot R_{q}}{\left(\sum_{q^{\prime}} \eta_{q^{\prime}}^{k} \eta_{q^{\prime}}^{l} R_{q^{\prime}}\right)}\left\{r_{q}^{k} \cdot r_{q}^{l}+\left(1-r_{q}^{k}\right) \cdot\left(1-r_{q}^{l}\right)\right\} .
\end{aligned}
$$

The term $r_{q}^{k} \cdot r_{q}^{l}$ is proportional to the number of events in which both charge tags are correct and the term $\left(1-r_{q}^{k}\right) \cdot\left(1-r_{q}^{l}\right)$ is proportional to the number of events in which both charge tags are wrong. Each term is been weighted by the flavour fraction of each tagging particle type in double tagged events.

As for the case of the branching fractions of the $Z^{0}$, one can construct a system of non-linear equations giving $\left(n^{2}+n\right) / 2$ constraints for $n$ tagging particle types, where $3 n$ unknown $r_{q}^{h}$ must be found before the asymmetries $A_{F B}(q)$ can be determined. Again the equation system involves products of the unknowns implying several ambiguous solutions. In accordance with the discussion in the previous section, one has to assume that in general the reliabilities $r_{q}^{h}$ are greater than 0.5 , since the leading hadron tends to carry the quantum numbers of the primary quark. Some exceptions are due to decays. Given that the charged kaons in events with primary down quarks have a charge sign opposite to that of the primary quark, $r_{d}^{K^{ \pm}}$is less than 0.5 . Note also that baryons do not carry the sign of the charge of the primary quark but the sign of the baryon quantum number, so that for the up-type quarks $r_{q}^{p}>0.5$ and for the down-type quarks $r_{q}^{p}<0.5$. Furthermore, one should be aware that the hadronisation contribution $H_{q}^{h}$ is completely charge symmetric.

If only charged pions, charged kaons, and protons are used, additional constraints are required. If the symmetry $\gamma_{u}=\gamma_{d}$ holds, then we can derive:

$$
\begin{aligned}
r_{u}^{\pi^{ \pm}} & =r_{d}^{\pi^{ \pm}}, \\
r_{s}^{\pi^{ \pm}} & =0.5, \quad \text { and } \\
r_{d}^{K^{ \pm}} & =\left(1-r_{u}^{K^{ \pm}}\right) \cdot \frac{\eta_{u}^{K^{ \pm}}}{\eta_{d}^{K^{ \pm}}} .
\end{aligned}
$$

As in the discussion above, the relations among reliabilities also may be broken if $\gamma_{u} \neq \gamma_{d}$. The breaking can be kept small for large $x_{c u t}$. 
Given that the widths $R_{q}$ and the various tagging efficiencies $\eta_{q}^{h}$ have been found, one can determine all of the light flavour reliabilities using the constraints from Eqs. $30-32$. In practice it is difficult to determine $r_{d}^{K^{ \pm}}$using the constraint from Eq. 32, since it is almost linear with the constraint one obtains using the measurement of double tagged events with charged pions and kaons.

\section{Possible Sources of Error}

Until now ideal particle identification capability has been assumed. In reality deficiencies in both the detection and the tagging procedures will affect the results and have to be taken into account. Some of the potential effects will be discussed in this section, which is intended to give a qualitative assessment of the potential biases. The final relevance of each of these issues depends on the details of the experimental analysis. Also of particular importance to this method is the validity of the assumed hadronisation symmetries. Some estimate of the size of the uncertainties of the branching fractions and asymmetries due to a possible breaking of the hadronisation symmetries will be given in Section 7 .

\subsection{Detector Effects}

The main experimental challenge to our proposal is the clear and unambiguous identification of the various particle species. Charged stable particles $\pi^{ \pm}, K^{ \pm}$, and $p, \bar{p}$ have been identified at LEP by $\mathrm{dE} / \mathrm{dx}$ measurements or Ring-imaging Cerenkov counters [13] up to the highest momenta. Naturally none of these samples is pure. Whereas the background from charged leptons and hyperons is small, the main experimental problem is the relative contaminations of the tagging particles among each other. For the basic equations (15) and (26-27) using single and double tagged events, it is important to know the compositions of the various measured samples.

The relation between apparent particle type $h^{\prime}$ and true particle type $h$ is given by some flow matrix, $\mathcal{E}_{h^{\prime}}^{h}$, such that

$$
N_{h^{\prime}}=\sum_{h} \mathcal{E}_{h^{\prime}}^{h} \cdot N_{h}
$$

The $K_{S}^{0}$ (and the $\Lambda$ ) should be largely decoupled from the stable charged hadrons because of the fundamentally different methods used to identify them. Once $\mathcal{E}_{h^{\prime}}^{h}$ is determined, the forward-backward asymmetries for measured samples $A_{F B}^{h^{\prime}}$ are related to the pure particle type asymmetries $A_{F B}^{h}$ by

$$
A_{F B}^{h^{\prime}}=\sum_{h} \mathcal{E}_{h^{\prime}}^{h} \cdot A_{F B}^{h} .
$$

Apart from reducing the discrimination power between the various flavours, some systematic uncertainties related both to the efficiency and purity are introduced.

Compared to charged particles, the efficiency for identifying neutral kaons is considerably smaller. Non-recoverable losses are the $50 \%$ of neutral kaons which decay into $K_{L}^{0}$ 
and also $K_{S}^{0}$ which decay into neutral pions. Simulation studies indicate that the smallness and the knowledge of the $K_{S}^{0}$ efficiency may be an experimentally limiting factor of this method.

\subsection{Correlations between Hemispheres}

The ideal relation $\eta_{q}^{k l}=\eta_{q}^{k} \cdot \eta_{q}^{l}$ for single and double tagged events is correct only if the tagging efficiencies in the two hemispheres are uncorrelated. In reality correlations exist due to the tagging procedure which modify the relation to $\left(\eta_{q}^{k l}\right)^{\text {effective }}=\rho \cdot \eta_{q}^{k} \cdot \eta_{q}^{l}=$ $\rho \cdot\left(\eta_{q}^{k l}\right)^{\text {ideal }}$, with $\rho \neq 1$. In the case of high $x_{p}$ tags for light flavours used in our method, there are dynamical as well as geometric correlations.

Collinear gluon radiation is one of the main reasons that fragmentation functions are spread out in energy. This is taken into account mostly by the measurement of the $\eta_{q}^{h}$. Some complications are due to hard, non-collinear gluon radiation, since this not only reduces the available energy of one quark, but induces a correlation between the hardness of the two primary quarks. The sign and size of the correlation depends on the emission angles and the energies of the gluons, both of which are quite well known from first principles of QCD. The net effect of gluon emission is to induce a positive correlation, $\rho>1$. The value of $\rho$ as a function of $x_{c u t}$ has been studied with the JETSET model for the four tagging hadrons and is found to be approximately $10 \%$ for $x_{\text {cut }} \approx 0.5$ (see Fig. 5). The detailed magnitude also depends somewhat on the shape of the fragmentation functions and is potentially different for different hadrons. Values of $\rho$ are also found to be consistent to within a few percent for the four tagging particle types. Further studies with the HERWIG model found similar results, indicating that the size of the correlation does not depend strongly on the hadronisation model used.

Geometric correlations can be introduced by the selection criteria to tag events. In an actual experiment one has to restrict the polar angle $\theta$ of the tagged particles or one might wish to restrict the angle $\theta_{12}$ between the two tagging particles in opposite hemispheres to be greater than some value. For Fig. 5 we required that the two tagging particles satisfy $\theta_{12}>90^{\circ}$. This requirement introduces a $\rho<1$ for low values of $x_{\text {cut }}$, while above $x_{c u t}>0.3$ the correlation is due almost entirely to gluon radiation. If this requirement is removed, the $\rho$ values tend to 1 as $x_{c u t} \rightarrow 0$. This is just one example of how selection criteria can introduce geometric correlations. The details will depend on the experimental situation and should be rather straightforward to estimate, but are beyond the scope of this paper.

One can determine $\rho$ from QCD generators after making sure that the measured and generated energy spectrum of the tags agree. Alternatively, one can attempt to determine a global $\rho$ from the data by introducing it as a free parameter in the non-linear equation system, thus avoiding any reliance on QCD models. Since $\rho$ is rather decoupled from the other free parameters, its uncertainty is found to have an only small impact on the final results. 


\subsection{Uncertainties in Hadronisation Assumptions}

To solve the equation system we have to invoke some assumptions about hadronisation symmetries. Although they are based on the rather fundamental SU(2) isospin symmetry and flavour independence of QCD, the symmetries may be broken by the less well understood aspects of hadronisation. This possible breaking is the basic uncertainty of the method. At the moment we see no unambiguous tests of the hadronisation assumptions. However, there are several important checks that can be carried out which should allow a reliable estimation of potential uncertainties.

One check is to ascertain if these relations depend on hadronisation parameters in models and on the models themselves. To this end we varied in the JETSET model the hardness of the fragmentation function (parameter PARJ (42) between 0.22 and 0.62 ), the fraction of strange quarks in the sea (parameter PARJ (2) between 0.27 and 0.36 ), the fraction of vector mesons (parameters PARJ(11) and PARJ (12) between 0.4 and 0.7 and between 0.2 and 0.6 , respectively), and assumed no production of $L=1$ mesons instead of about $16 \%$ (parameters PARJ(13-17)). In all cases we find no statistically significant deviation for the relations of Eqs. $20-23$, supporting our estimate of a possible breaking of at most $1-2 \%$. The relations were also checked with the HERWIG model, which employs a cluster decay mechanism [18] to simulate hadronisation. An interpretation of the results, however, is complicated since this model does not preserve isospin symmetry due to technical reasons [25]. As a result, only the relation of Eq. 20 can be meaningfully checked and is found to hold, whereas relations between neutral and charged kaons are broken by less than 3\%. As anticipated by the discussion in Section 4.3, the last two candidate relations (Eqs. $24-25$ ) are less certain. The variation of the fragmentation parameters in JETSET implies a change of $\sim 3-4 \%$ in the relation of Eq. 24 and $\sim 12 \%$ for Eq. 25.

Another test is the use of the fairly complete and precise measurements of the production of hadron resonances in jets at LEP [22]. Since hadron resonance decays are well known, these measurements can be used to estimate any possible breaking due to the decay contribution $Z_{q}^{h}$. No direct measurements exist for the size of the hadronisation contribution $H_{q}^{h}$. However, a global test of its effects can be performed. One consequence of the charge symmetric $H_{q}^{h}$ contribution is to dilute the reliability. Therefore, apart from some known contributions due to decays, the dependence of the various $r_{q}^{h}$ on $x_{c u t}$ will indicate the size of the hadronisation component. Note that to high accuracy, this hadronisation component should be independent of the primary quark flavour. The measurement of the global hadronisation contribution will allow a reasonable estimate of its effect on symmetry breaking.

\section{$7 \quad$ A Model of a Measurement}

The method outlined in this paper was tested on a sample of approximately 15 million JETSET 7.4 hadronic $Z^{0}$ decay events. The size of the event sample is some factor four larger than what is available at an individual LEP experiment, but is large enough 
so that any potential systematic problems with the model are not hidden by statistical fluctuations. No detector effects were considered. The numbers of high $x_{p}>0.4 \pi^{ \pm}, K^{ \pm}$, $K_{S}^{0}$, and $p(\bar{p})$, which represent most of the particle types for which reasonably statistically accurate measurements in both single and double tags can be made, are given in Table 1.

\begin{tabular}{|c||c|c|c|c|c|}
\hline \multicolumn{1}{|c|}{ hadron type } & single tags & \multicolumn{4}{|c|}{ double tags } \\
& & $\pi$ & $K^{ \pm}$ & $p(\bar{p})$ & $K_{S}^{0}$ \\
\hline \hline$\pi$ & 1356405 & 48367 & 32527 & 20479 & 16421 \\
\hline$K^{ \pm}$ & 835868 & & 21870 & 11937 & 21388 \\
\hline$p(\bar{p})$ & 322956 & & & 2946 & 5617 \\
\hline$K_{S}^{0}$ & 404819 & & & & 5325 \\
\hline
\end{tabular}

Table 1: Number of single and double tags as expected by the JETSET model for 15 million hadronic $Z^{0}$ decays.

The system of Eq. 15 for $\pi^{ \pm}, K^{ \pm}, K_{S}^{0}$, and $p(\bar{p})$ was solved using $x_{\text {cut }}=0.4$ for the following free parameters: the unknown light flavour branching fraction $R_{s}=R_{d}$, a global double tag efficiency correction $\rho$, and the several unknown light flavour efficiencies $\eta_{q}^{h}$. Given that the heavy flavour branching fractions of the $Z^{0}$ are known, $R_{u}$ is not an independent variable but is constrained by $R_{u}=1-\left(R_{d}+R_{s}\right)-R_{c}-R_{b}$. For hadronisation symmetries, the relations of Eqs. 20-23 were assumed. All of the heavy flavour fraction branching fractions of the $Z^{0}$ were fixed to their JETSET values and the heavy flavour $\eta_{c}^{h}$ and $\eta_{b}^{h}$ were taken according to the model with appropriate uncertainties.

The results for $R_{d}=R_{s}$ are given in Table 2 for several values of $x_{c u t}$. It is accurately determined, with a statistical error of some two percent for $x_{c u t}=0.4$. Obviously the statistical accuracy decreases with increasing $x_{c u t}$. On the other hand, low values of $x_{c u t} \leq 0.3$ lead to an apparent bias which can be understood from the preceding discussion on hadronisation symmetries. In addition to these criteria, the optimal $x_{c u t}$ in an actual experiment will depend on the knowledge of the heavy quark background, which decreases with increasing $x_{c u t}$, and the potential of disentangling the various hadron species, which often degrades with higher energies. Therefore, the optimal $x_{c u t}$ will depend on the experimental details.

\begin{tabular}{|c|c|}
\hline$x_{\text {cut }}$ & $\begin{array}{c}\text { method result } \\
R_{d}=R_{s}\end{array}$ \\
\hline$\overline{00.2}$ & $0.263 \pm 0.006$ \\
\hline 0.3 & $0.232 \pm 0.003$ \\
\hline 0.4 & $0.223 \pm 0.004$ \\
\hline 0.5 & $0.221 \pm 0.005$ \\
\hline 0.6 & $0.220 \pm 0.006$ \\
\hline
\end{tabular}

Table 2: Fit results (with statistical errors only) of $R_{d}=R_{s}$ for various values of $x_{\text {cut }}$. The input value for $R_{d}=R_{s}$ is 0.220 in JETSET. Note that the results are not statistically independent.

To obtain the forward-backward asymmetries of the light quarks, Eqs. 26-27 were used to determine the pion, kaon, and proton reliabilities, assuming the relations in Eqs. 30-32. 
Almost all of the reliabilities were determined with typical relative statistical accuracies of a few percent. One noticeable exception is $r_{d}^{K^{ \pm}}$, which has a Monte Carlo input value of $r_{d}^{K^{ \pm}}=0.20$, but for which the method finds $0.03 \pm 0.10$. The problem was found to be a near linearity between Eq. 32 and the constraint on $r_{d}^{K^{ \pm}}$and $r_{u}^{K^{ \pm}}$from the measurement of double tagged pion-kaon events. In order to overcome this problem, $r_{d}^{K^{ \pm}}$was fixed to 0.20 with a \pm 0.10 error. Using these reliabilities and constraints, the method yields forward-backward asymmetries of the combined down and strange quarks and the up quark as listed in Table 3. Note that in contrast to the requirement of the sum of the $R_{q}$ to be one, the asymmetries of the various flavours are not correlated by some overall constraint. As can be seen from Table 3 the asymmetries are reproduced by our method.

\begin{tabular}{|c|c|c|}
\hline Observable & input & method result \\
\hline \hline$A_{F B}(d, s)$ & $10.0 \%$ & $9.41 \pm \mathbf{0 . 2 4 \%}$ \\
\hline$A_{F B}(u)$ & $7.1 \%$ & $5.61 \pm \mathbf{0 . 4 6 \%}$ \\
\hline
\end{tabular}

\begin{tabular}{|c|c|c|}
\hline & $A_{F B}(d, s)$ & $A_{F B}(u)$ \\
\hline \hline$A_{F B}(d, s)$ & 1.000 & 0.529 \\
\hline$A_{F B}(u)$ & & 1.000 \\
\hline
\end{tabular}

Table 3: Input and fit results (with statistical errors only) for the light flavour forwardbackward asymmetries, with the correlation matrix.

As yet only statistical uncertainties have been considered. It was frequently mentioned previously that the hadronisation symmetries are particularly important for this method. As can be observed in Fig. 3, no statistically significant deviations of more than $2 \%$ are observed for the $x_{c u t}$ range considered in our analysis, even when variations in the JETSET fragmentation parameters are made. The impact on $R_{d}=R_{s}$ of allowing the hadronisation symmetries to be broken by $2 \%$ is given in Table 4 . The largest sensitivity of the result is due to the relation $\eta_{s}^{K^{0}}=\eta_{s}^{K^{ \pm}}$, which in effect separates the up and down contributions. The total fractional uncertainty inherent to this method is therefore $\sim 5 \%$. In an experimental analysis, there will of course be other experimental uncertainties, such those due to the precision with which the heavy flavour contributions can be measured.

Concerning the asymmetries, uncertainties in the reliability relations have to be considered. As can be seen from Table 5, these relations affect the quark flavours in different ways and all together lead to an absolute systematic uncertainty of $\delta A_{F B}(q) \sim 1 \%$. Thus, we obtain a total uncertainty of $\sim 1.5 \%$ for both $A_{F B}(d)=A_{F B}(s)$ and $A_{F B}(u)$. An additional uncertainty is due to the limited knowledge of $R_{d}=R_{s}$ and $R_{u}$, which affects $A_{F B}(u)$ much more strongly than $A_{F B}(d)=A_{F B}(s)$.

\begin{tabular}{|c|c|c|}
\hline relation & variation & $\delta R_{s}=\delta R_{d}$ \\
\hline \hline$\eta_{d}^{\pi} / \eta_{u}^{\pi}$ & $1.00 \pm \mathbf{0 . 0 2}$ & $\pm \mathbf{0 . 0 0 1}$ \\
\hline$\eta_{d}^{K^{0}} / \eta_{u}^{K^{ \pm}}, \eta_{u}^{K^{0}} / \eta_{d}^{K^{ \pm}}$ & $1.00 \pm \mathbf{0 . 0 2}$ & $\mp \mathbf{0 . 0 0 5}$ \\
\hline$\eta_{s}^{K^{0}} / \eta_{s}^{K^{ \pm}}$ & $1.00 \pm \mathbf{0 . 0 2}$ & $\mp \mathbf{0 . 0 1 2}$ \\
\hline
\end{tabular}

Table 4: Uncertainties in $R_{d}=R_{s}$ due to assumptions about hadronisation. 


\begin{tabular}{|c|c|c|c|}
\hline relation & variation & $\begin{array}{c}\delta A_{F B}(d) \\
=\delta A_{F B}(s)\end{array}$ & $\delta A_{F B}(u)$ \\
\hline \hline$r_{d}^{K}$ & $\mathbf{0 . 2 0} \pm \mathbf{0 . 1 0}$ & $\mp \mathbf{0 . 5 7 \%}$ & $\mp \mathbf{0 . 1 2 \%}$ \\
\hline$r_{u}^{\pi} / r_{d}^{\pi}$ & $1.00 \pm \mathbf{0 . 0 5}$ & $\mp \mathbf{0 . 2 5 \%}$ & $\mp 1.11 \%$ \\
\hline$r_{s}^{\pi}$ & $\mathbf{0 . 5 0} \pm \mathbf{0 . 0 5}$ & $\mp \mathbf{0 . 3 4 \%}$ & $\mp \mathbf{0 . 4 5 \%}$ \\
\hline \hline$\delta R_{d}=\delta R_{s}$ & $\mathbf{0 . 2 2 3 \pm \mathbf { 0 . 0 1 1 }}$ & $\pm \mathbf{0 . 0 3 \%}$ & $\pm 1.22 \%$ \\
\hline
\end{tabular}

Table 5: Uncertainties in the forward-backward asymmetries due to assumptions about reliabilities and $R_{d}=R_{s}$.

In applying the method to Monte Carlo events, we have used so far only the hadronisation relations which follow from $\mathrm{SU}(2)$ isospin symmetry and the flavour independence of QCD. As discussed in Section 4.3, a phenomenological study of the JETSET model suggests that additional symmetry relations might be used. Including those (Eqs. $24-25$ ) in the analysis allows one to disentangle the electroweak observables of all individual quark flavours. The obtainable precision depends on the extent to which these relations are allowed to be broken. Assuming, for example, a relative precision of $5 \%$ for the relation of Eq. 24 and $12 \%$ for Eq. 25, we estimate that the method leads to $\delta R_{q} / R_{q} \sim 12-15 \%$. Although we have not made an exhaustive search for new relations, it is possible that if more relations are found, this uncertainty can be reduced.

\section{The Knowledge of the $\eta_{q}^{h}$}

Although in this article we focus on the measurement of electroweak couplings of light flavours to the $Z^{0}$, our proposal reaches further. Due to the high statistics and the possibility of double tags, $Z^{0}$ decays offer, maybe uniquely, the chance to measure efficiencies and purities of the individual light flavour species. Based on the example of a measurement given in the previous section, the $\eta_{q}^{h}$ are determined with relative errors of about $1 \%$. Assuming Standard Model values for the $R_{q}$, almost a factor of two better precision for $\eta_{q}^{h}$ for most flavours and hadron types can be obtained. Such knowledge may be of interest for QCD studies but also for measurements of the decay properties of heavy particles other than the $Z^{0}$, such as the $W^{ \pm}$boson and the top quark. After the effects of the differing kinematics of the events and QCD scaling violations of the fragmentation functions have been taken into account, the $\eta_{q}^{h}$ 's obtained from $Z^{0}$ decays may be used.

Given proper particle identification and high enough statistics, experiments may be able to determine the decay branching ratios of $W^{ \pm}$bosons and top quarks, for example. At the proposed linear $e^{+} e^{-}$collider with a centre-of-mass energy of $400-500 \mathrm{GeV}$, some $600,000 W^{ \pm}$bosons should be available [26]. With the tagging methods discussed, the relative decay modes into various quarks species may be measurable by comparing decays with leading $K^{+} K^{+}, K^{+} \pi^{+}$, or $\pi^{+} \pi^{+}$, for instance. The attainable precision may in some cases be comparable to the current knowledge of the Cabibbo-Kobayashi-Maskawa mixing matrix elements. However, such a measurement would not depend on models of hadron decays, as is frequently the case in today's analyses. Similarly, some 30,000 
semileptonic top decays are expected at a linear collider, which implies some $300-600$ decays of $t \rightarrow s+X$. With a well understood strange quark tag, these may also be measurable.

Concerning QCD studies, there are several instances in which light flavour tags could be useful at LEP, such as in measurements of the flavour independence of $\alpha_{s}$ and the determination of gluon versus quark fragmentation functions. Other areas of interest could be the study of hadronisation models. For example, the HERWIG model invokes cluster decays which, in their most simple version, decay isotropically. As a result the charge and flavour correlations are significantly smaller than in the string model where the leading particle retains the original flavour. Another example is a test of the diquark model [27] of baryon production. Due to Fermi statistics, this model implies the production of $(u u)$ diquarks is suppressed relative to $(u d)$ diquarks. Thus protons produced directly from primary quarks should be due to up quarks only.

\section{Conclusions}

In this paper it has been shown that by taking advantage of the high statistics of LEP and using hadronisation symmetries based on $\mathrm{SU}(2)$ isospin symmetry and the flavour independence of QCD, insight into the production and structure of hadronic $Z^{0}$ decays to light flavours can be obtained. The method avoids reliance on detailed model assumptions such as the shape of the fragmentation function or the fraction of strange quarks in the QCD sea. We emphasised in this article the possibilities of determining electroweak properties of light flavours. With the statistics presently available to each of the LEP experiments, we estimate that the inherent precision of the method is $\delta\left(R_{d}=R_{s}\right) /\left(R_{d}=R_{s}\right) \sim 5 \%$ and $\delta A_{F B}(d=s) \sim \delta A_{F B}(u) \sim 0.010-\mathbf{0 . 0 1 5}$ for the corresponding asymmetries. The final experimental precision will also depend on the ability of an experiment to identify high momentum particles and to measure the heavy flavour contributions. Somewhat more model dependent hadronisation symmetries can be introduced that allow the determination of the branching fractions of the individual light flavours. To achieve good precision measurements, however, several hadronisation relations of reasonable precision must be used.

The complete set of these measurements will imply rather strong constraints on possible deviations from the Standard Model. How strong these will be in the end is a question beyond this paper. In any case, our paper shows that it is possible to derive rather secure information on individual light flavours from LEP. Such an endeavour becomes all the more relevant in the light of the ' $R_{b} R_{c}$ ' crisis. The possible measurements in the light flavour sector may be an important complement to the high precision tests of the electroweak Standard Model achieved at LEP. 


\section{Acknowledgements}

In developing the method we profited from discussions with a large number of people. We are particularly indebted to M. Dallavalle, C. Grandi, and M. Hauschild. We would also like to thank M. Dittmar, P. Schenk, T. Sjöstrand, and D. Wegener for their careful reading of the draft and for their very helpful comments. 


\section{References}

[1] S.L. Glashow, J. Iliopoulos, and L. Maiani, Phys. Rev. D2 (1970) 1285;

S. Weinberg, Phys. Rev. Lett. 19 (1967) 1264;

A. Salam, Elementary Particle Theory, Ed. N. Svartholm (Almquist and Wiksells, Stockholm, 1969) pp. 367.

[2] The LEP Collaborations ALEPH, DELPHI, L3, and OPAL, and the LEP Electroweak Working Group, CERN-PPE/95-172.

[3] For a summary of recent results, see:

H. Evans, Physics of the $\tau$ Lepton, to be published in the Proceedings of the Lake Louise Winter Institute (1996), Lake Louise, Canada.

[4] The results from ALEPH, DELPHI, L3, OPAL, and SLD are, for example, summarised in:

M. Schmelling, Phys. Scr. 51 (1995) 683.

[5] Among the many proposals see, for example:

E. Ma, Phys. Rev. D53 (1996) 2276;

G. Bhattacharyya et al., CERN-TH/95-326, FISIST/14-95/CFIF, NTUTH-95-11;

T. Yoshikawa, HUPD-9528.

[6] See, for example:

P. Chiappetta et al., PM/96-05, CPT-96/P.3304;

G. Altarelli et al., CERN-TH/96-20;

K.S. Babu et al., IASSNS-HEP-96/20;

and references in [7].

[7] K. Agashe et al., LBL-38569;

V. Barger, K. Cheung, and P. Langacker, MADPH-96-936, UPR-0696T, UTEXASHEP-96-2, DOE-ER-40757-078.

[8] P. Mättig and W. Zeuner, Z. Phys. C52 (1991) 37;

The LEP results from ALEPH, DELPHI, L3, and OPAL are combined in:

P. Mättig, Single Photon and Radiative Events at LEP, CERN-PPE/95-81;

See also: J.L. Feng et al., Phys. Rev. Lett. 76 (1996) 3259.

[9] DELPHI Collaboration, P. Abreu et al., Z. Phys. C67 (1995) 1.

[10] T. Sjöstrand, Comp. Phys. Comm. 39 (1986) 347;

T. Sjöstrand and M. Bengtsson, Comp. Phys. Comm. 43 (1987) 367.

[11] For an early outline of the method see, for example:

P. Mättig, A High Luminosity LEP as a B-Factory, CERN-EP/90-71.

LEP measurements using double tagged events based on lifetime information:

ALEPH Collaboration, D. Buskulic et al., Phys. Lett. B313 (1993) 535;

DELPHI Collaboration, P. Abreu et al., CERN-PPE/96-15;

OPAL Collaboration, R. Akers et al., Z. Phys. C65 (1995) 17.

[12] HRS Collaboration, P. Kesten et al., Phys. Lett. B161 (1985) 412. 
[13] ALEPH Collaboration, D. Buskulic et al., Z. Phys. C66 (1995) 355;

DELPHI Collaboration, P. Abreu et al., Nucl. Phys. B444 (1995) 3;

OPAL Collaboration, R. Akers et al., Z. Phys. C63 (1994) 197.

[14] C. Grandi, Studio dei Decadimenti della $Z^{0}$ in Quark Leggeri col Rivelatore OPAL al $L E P$, Ph.D. thesis, Universitá degli Studi di Bologna (Italy), February 1996, unpublished.

[15] OPAL Collaboration, R. Akers et al., Z. Phys. C67 (1995) 27.

[16] Particle Data Group, L. Montanet et al., Phys. Rev. D50 (1994) 1173.

[17] Summary of experiments at PETRA, PEP, and CESR in:

P. Mättig, Phys. Rep. 177 (1989) 141.

[18] S. Wolfram, in Proceedings of the $15^{\text {th }}$ Recontre de Moriond, Ed. J. Tran Thanh Van (1980).

[19] G. Marchesini et al., Comp. Phys. Comm. 76 (1992) 464.

[20] B. Andersson, G. Gustafson, G. Ingelman, and T. Sjöstrand, Phys. Rep. 97 (1983) 33.

[21] OPAL Collaboration, R. Akers et al., Phys. Lett. B353 (1995) 595.

[22] For a recent compilation of measured hadron rates measured by ALEPH, DELPHI, L3, and OPAL see:

G.D. Lafferty, P.I. Reeves, and M.R. Whalley, J. Phys G: Nucl. Part. Phys. 21 (1995) A1.

[23] G. Bocquet et al., Phys. Lett. B366 (1996) 447.

[24] B. Andersson, G. Gustafson, and B. Soderberg, Z. Phys. C20 (1983) 317.

[25] T. Sjöstrand, QCD Generators, in "Z Physics at LEP", Eds. G Altarelli, R. Kleiss, and C. Verzegnassi, CERN/89-08 (1989), Vol. 3, p. 250.

[26] See, for example:

B.H. Wiik, in Proceedings of the $2^{\text {nd }}$ International Workshop on Physics and Experiments with Linear $e^{+} e^{-}$Colliders (1993), Waikoloa, Hawaii.

[27] For a review, see:

M. Anselmino et al., Rev. Mod. Phys. 65 (1993) 1199. 


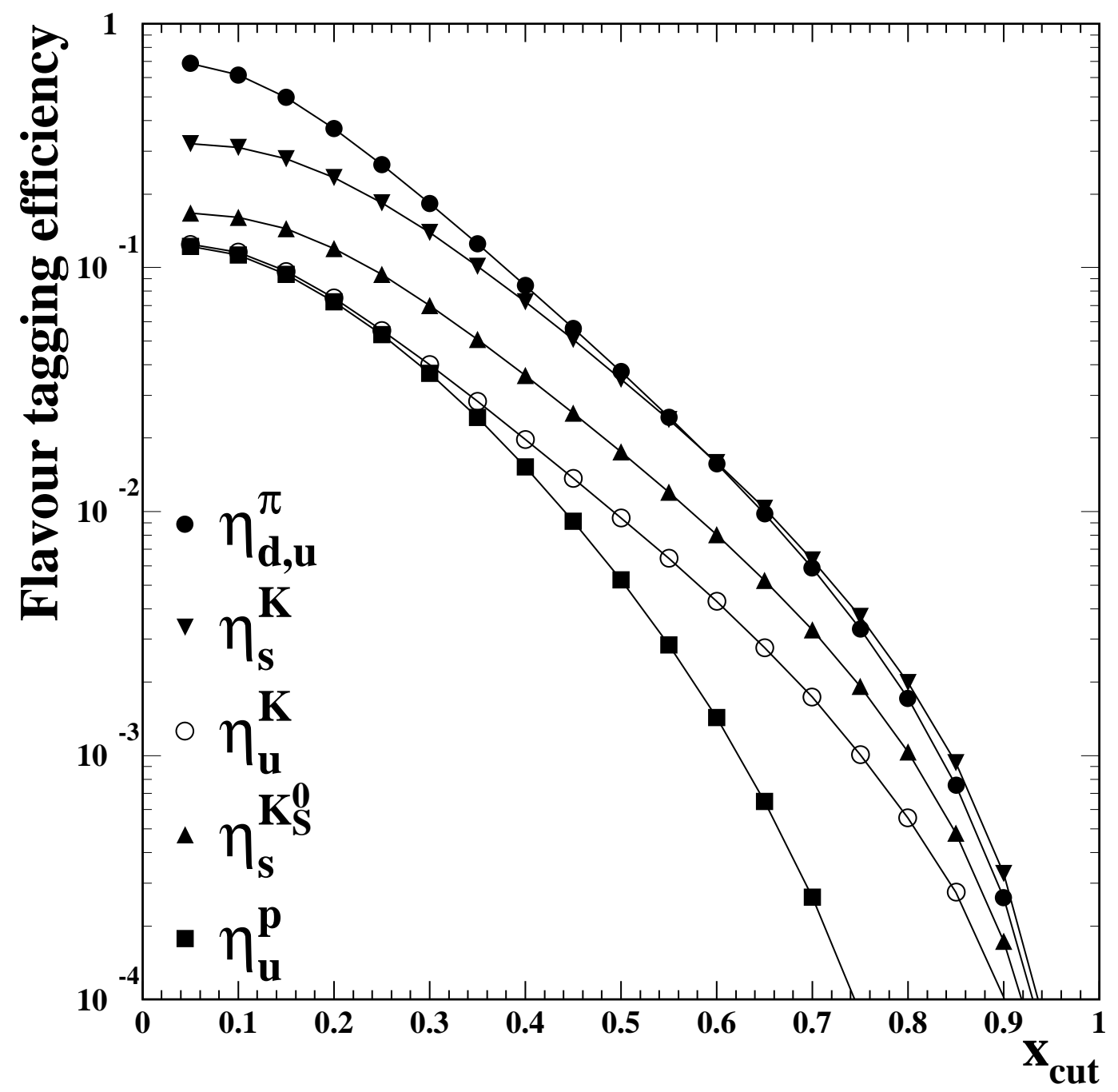

Figure 1: Tagging efficiencies $\eta_{q}^{h}$ per event hemisphere for various quark flavours $q$ and different tagging particle types $h$ as a function of the minimum scaled particle momentum $x_{p}=2 p_{h} / E_{c m}>x_{c u t}$. 


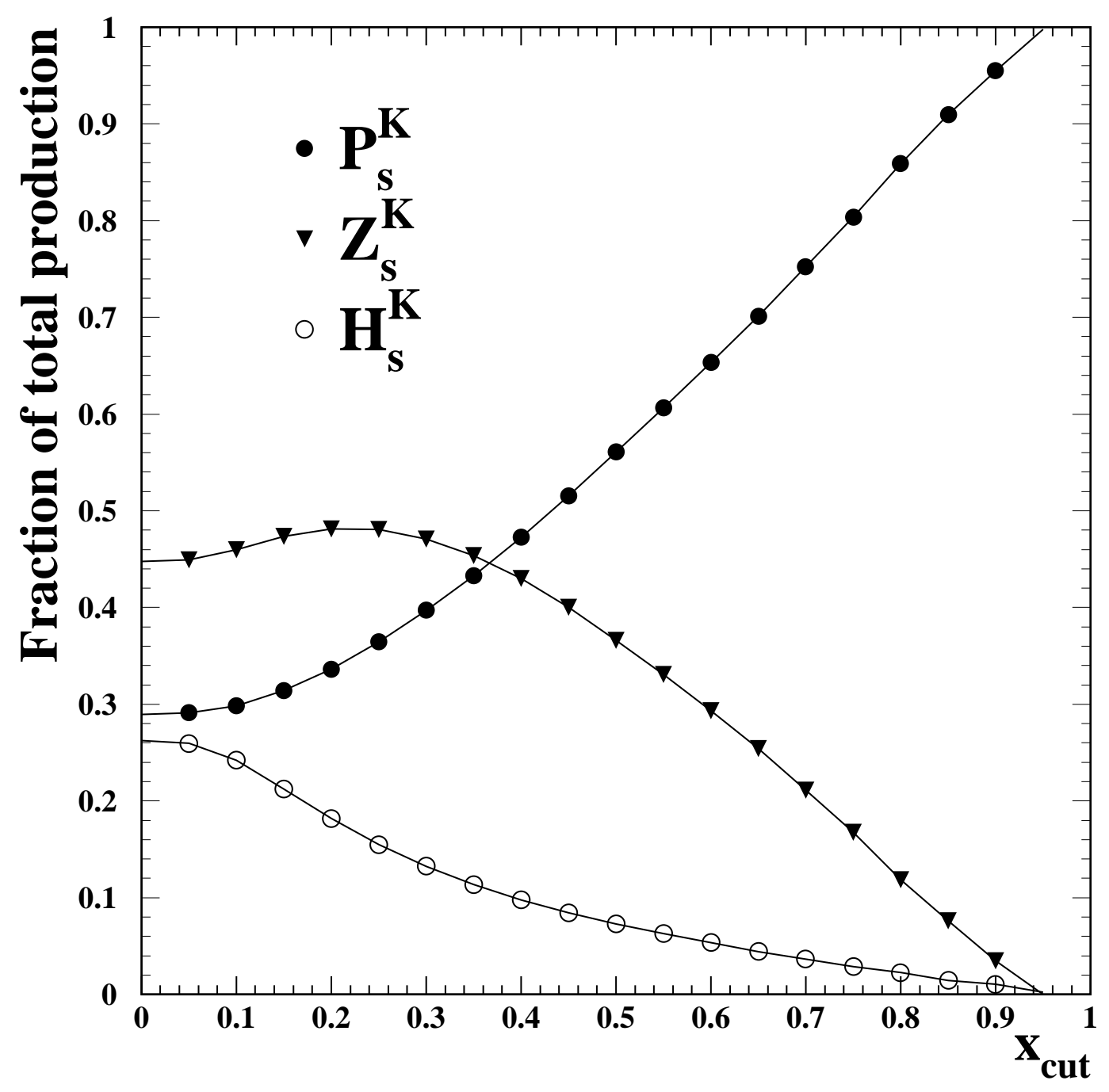

Figure 2: Relative contributions of primary production $P_{s}^{K}$, decays $Z_{s}^{K}$, and hadronisation production $H_{s}^{K}$ of charged kaons in $s$ quark events as a function of $x_{c u t}$. The curves are to guide the eye. 

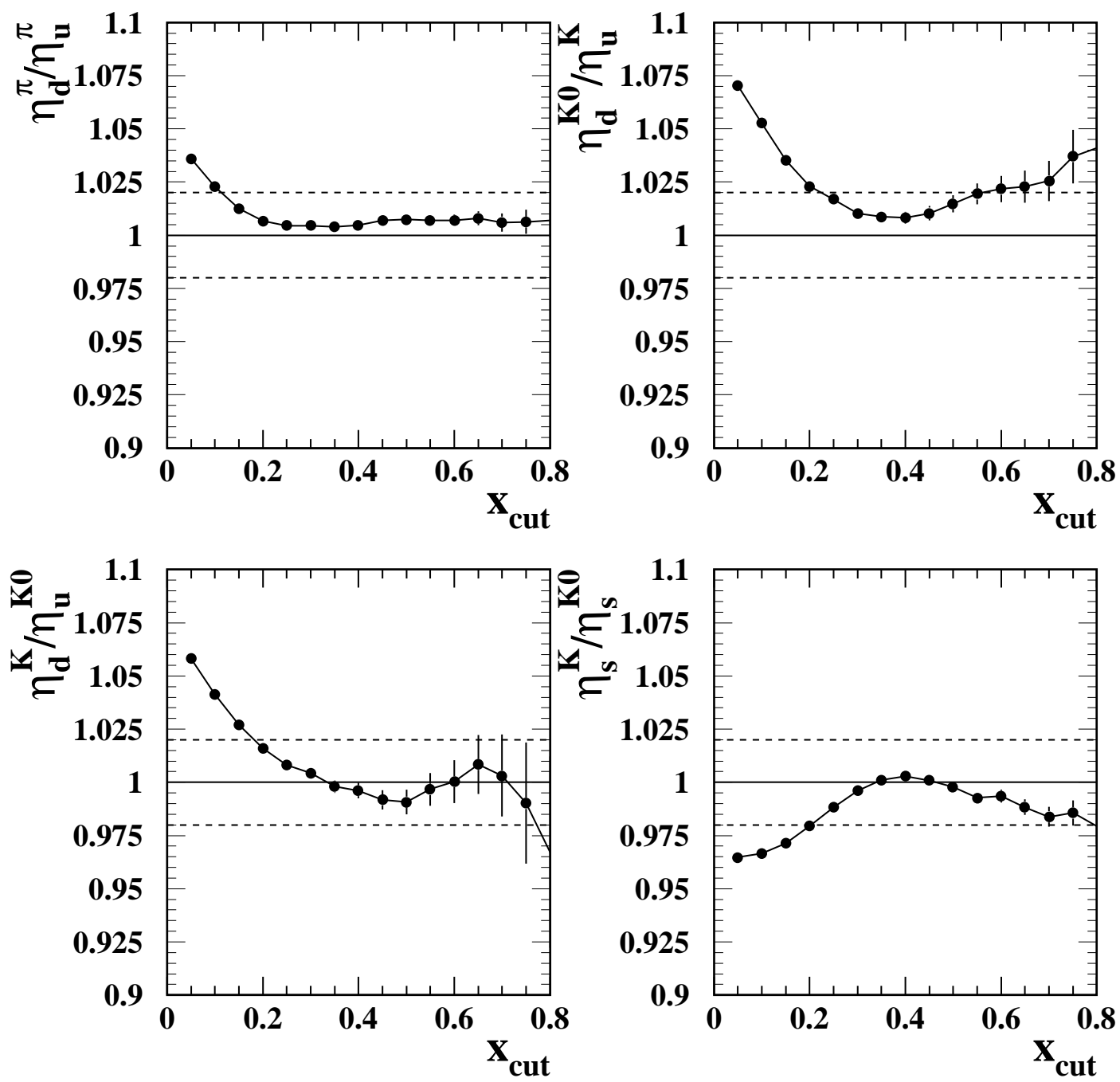

Figure 3: Examples of the breaking of some of the hadronisation relations as a function of $x_{c u t}$. Note that the various results as a function of $x_{c u t}$ are correlated. The bands indicated by the dashed lines correspond to a breaking of the relations by $\pm 2 \%$. 

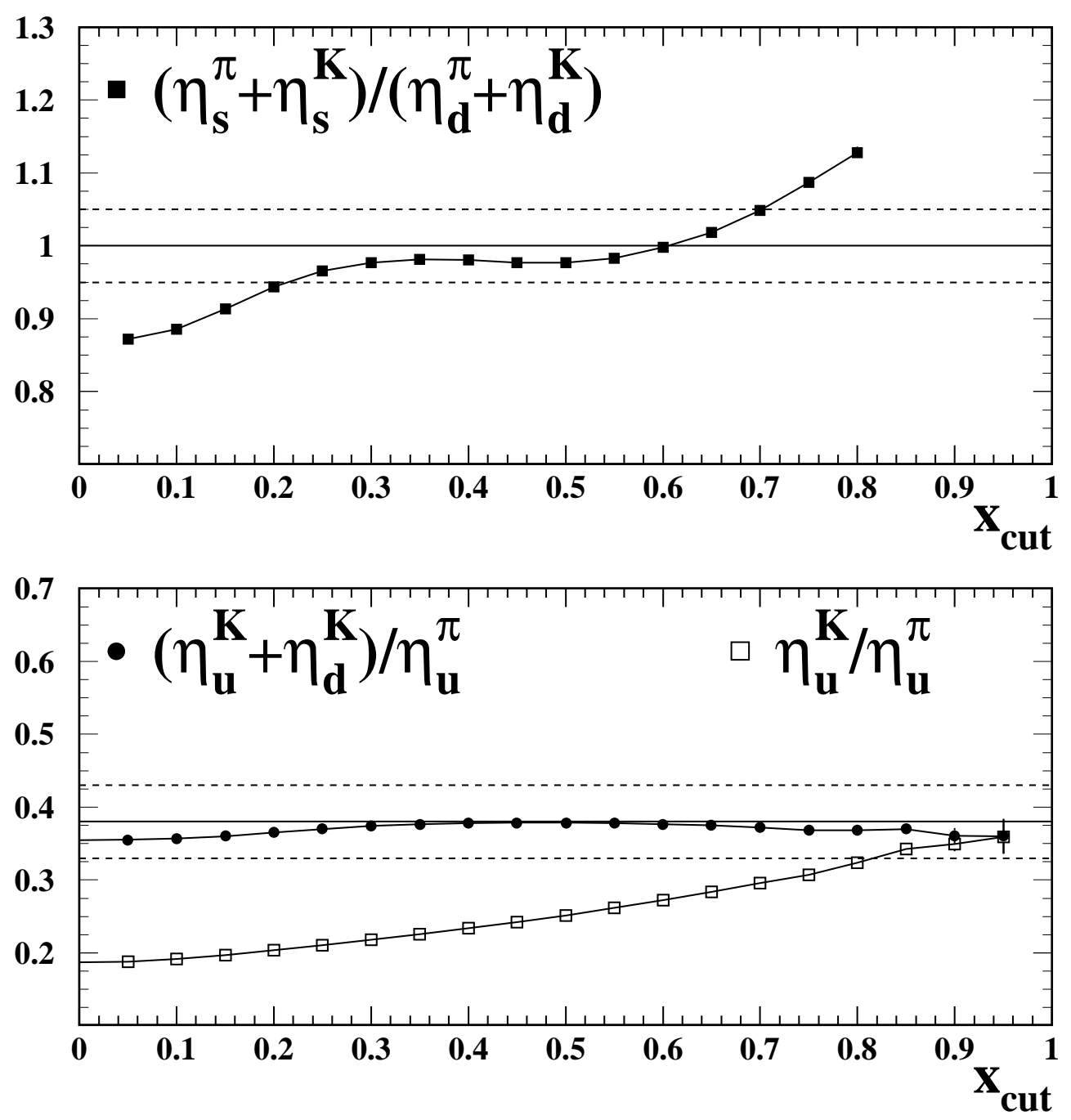

Figure 4: Two supplementary hadronisation symmetries. These relations are somewhat more model dependent than those in Fig. 3 (see text). The dashed lines indicate deviations of \pm 0.05 from 1.00 and 0.38 , respectively. 


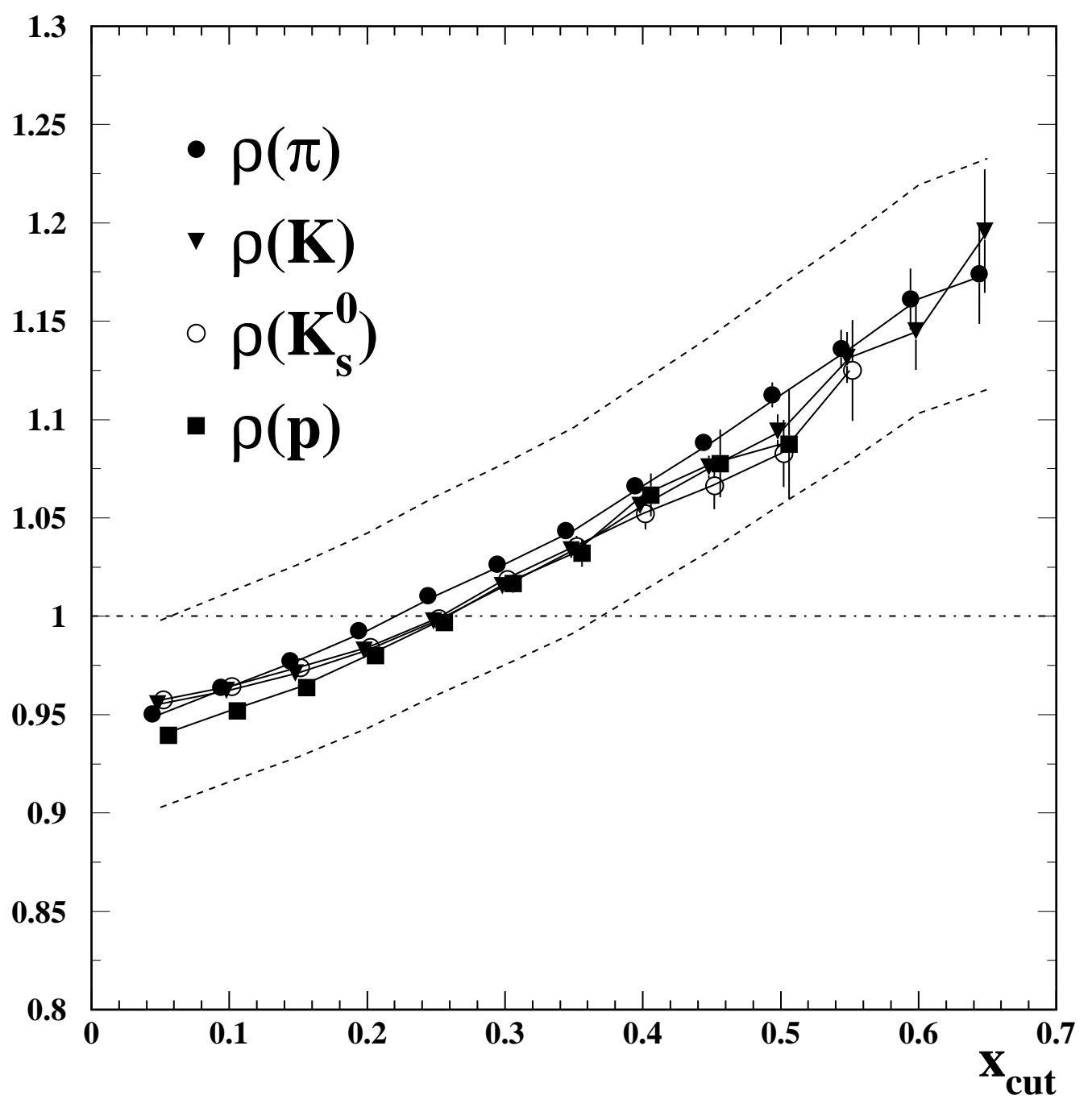

Figure 5: The dependence of $\rho(h)$, which measures the strength of the correlation between the tagging efficiencies in opposite hemispheres, as a function of $x_{c u t}$ for different tagging particle types, $h$. A cut on the angle between the two tagging particles in double tagged events $\theta_{12}>90^{\circ}$ was made. The dashed lines show $\pm 5 \%$ around $\rho(\pi)$. 